\title{
Optical properties of the nitrogen vacancy in AIN epilayers
}

\author{
N. Nepal, K. B. Nam, M. L. Nakarmi, J. Y. Lin, and H. X. Jiang ${ }^{a)}$ \\ Department of Physics, Kansas State University, Manhattan, Kansas 66502-2601 \\ J. M. Zavada \\ U.S. Army Research Office, Durham, North Carolina 27709
}

R. G. Wilson

Stevenson Ranch, California 91381

(Received 30 September 2003; accepted 22 December 2003)

\begin{abstract}
AlN epilayers grown by metalorganic chemical vapor deposition were implanted with cobalt ions and studied by deep UV photoluminescence (PL). A PL emission peak at $5.87 \mathrm{eV}$ (at $10 \mathrm{~K}$ ) was observed for the Co-implanted AIN epilayers, which was absent in as-grown AlN epilayers. Temperature dependence of the PL intensity of the $5.87 \mathrm{eV}$ emission line revealed an ion-implantation induced defect with energy level of about $260 \mathrm{meV}$ below the conduction band. The $5.87 \mathrm{eV}$ emission line is believed due to a band-to-impurity transition involving the nitrogen vacancy $\left(V_{\mathrm{N}}\right)$ in ion-implanted AlN. The experimentally determined energy level of the nitrogen vacancy is in reasonable agreement with the calculated value of $300 \mathrm{meV}$. From the band-to-impurity transition involving $V_{\mathrm{N}}$, we have deduced the energy band gap of AlN to be 6.13 $\mathrm{eV}$, which is consistent with our previous result. Our results suggest that nitrogen vacancies in AlN cannot make any significant contribution to the $n$-type conductivity due to the large binding energy as well as the large formation energy. (C) 2004 American Institute of Physics.
\end{abstract}

[DOI: 10.1063/1.1648137]

Aluminum nitride $(\mathrm{AlN})$ has attracted much interest due to its applications in UV light emitters and other optoelectronic devices. It has high mechanical hardness, high thermal conductivity, large dielectric constant, and high resistance to harsh environments. ${ }^{1}$ It may also be promising in the emerging field of spintronics, due to its predicted high curie temperature $\left(T_{C}\right)$ when doped with particular transition metals. ${ }^{2,3}$ For further applications of AlN, it is very important to understand its fundamental properties. For optoelectronic device applications based on AlN, understanding the properties of native defects and impurities is crucial; particularly their charge states and energy levels.

As-grown $\mathrm{GaN}$ is usually $n$ type; however, as-grown AlN is highly resistive and $n$-type conductivity is very difficult to obtain. For many years, it was believed that nitrogen vacancies $\left(V_{\mathrm{N}}\right)$ are the cause of the high background $n$-type conductivity in unintentionally doped GaN. ${ }^{4}$ First principle calculations indicate that the isolated nitrogen vacancy in GaN has large formation energy and can be excluded as the source of $n$-type conductivity. ${ }^{5}$ More recently, nitrogen vacancies have been observed in electron-irradiated GaN epilayers and the energy level of $V_{\mathrm{N}}$ in $\mathrm{GaN}$ was determined to be around $25 \mathrm{meV}^{6}$ This is close to a value of $30 \mathrm{meV}$ obtained from a tight-binding calculation ${ }^{7}$ and is consistent with the value obtained from the effective-mass theory without central-cell correction. ${ }^{8}$ While the properties of $V_{\mathrm{N}}$ in $\mathrm{GaN}$ are relatively well understood now, little is known about $V_{\mathrm{N}}$ in AlN. Previous theoretical investigation showed that $V_{\mathrm{N}}$ is a shallow donor with an energy level of about 300 meV. ${ }^{7}$ While deep levels offer preferential recombination

${ }^{a)}$ Electronic mail: jiang@phys.ksu.edu routes, they are frequently nonradiative and thus inimical to luminescent devices.

In this letter, we report deep UV photoluminescence (PL) results of cobalt (Co) implanted AIN epilayers, from which we have deduced the energy band diagram for the $V_{\mathrm{N}}$ levels in GaN and AlN. The 1- $\mu$ m-thick AlN epilayers were grown by metalorganic chemical vapor deposition (MOCVD) on sapphire (0001) substrates with low temperature AlN nucleation layers. ${ }^{9}$ Trimethylaluminum and blue ammonia $\left(\mathrm{NH}_{3}\right)$ were used as $\mathrm{Al}$ and nitrogen sources. $\mathrm{Co}^{+}$ ions were implanted in AlN at an energy of $250 \mathrm{keV}$ and with a fixed dose of $3 \times 10^{16} \mathrm{~cm}^{-2}$ as described elsewhere 3 and Co-implanted AIN epilayers were then thermally annealed in nitrogen ambient at various temperatures up to $1325^{\circ} \mathrm{C}$ for $15 \mathrm{~min}$. Structural characterization was done through high-resolution x-ray diffraction (XRD) measurements. Full width at half maximum of XRD rocking curve of (0002) peak of the as-grown AlN epilayers was around 400 arc sec, which increased by about $30 \%$ for Co-implanted AlN epilayers, but the peak position didn't change. Deep UV PL spectroscopy was employed to investigate the optical properties of the Co-implanted samples. The PL system consists of a frequency quadrupled $100 \mathrm{fs}$ Ti:sapphire laser having an average power of $3 \mathrm{~mW}$. The excitation photon energy was set at $6.28 \mathrm{eV}$ with a repetition rate of $76 \mathrm{MHz}$. A $1.3 \mathrm{~m}$ monochromator and a streak camera, with a detection capability ranging from 185 to $800 \mathrm{~nm}$ and a time resolution of 2 ps, were used. ${ }^{10}$

Figure 1 compares the low temperature $(10 \mathrm{~K}) \mathrm{PL}$ spectra of (a) as-grown and (b) Co-implanted AlN epilayers. The arrows indicate the peak positions of the spectra. The asgrown epilayer exhibits a strong band edge emission line at $6.05 \mathrm{eV}$ and its $\mathrm{LO}$ phonon replicas at $5.94 \mathrm{eV}$ (1 LO) and 


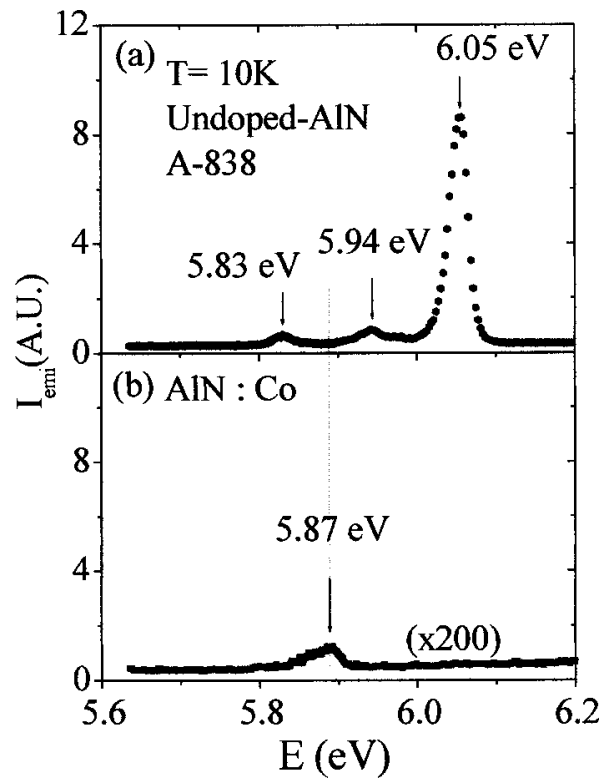

FIG. 1. PL spectra of (a) as-grown AIN and (b) Co-implanted AlN epilayer measured at $10 \mathrm{~K}$. For the Co-implanted AlN epilayer, the band edge transition at $6.05 \mathrm{eV}$ disappears and an emission line is observed at $5.87 \mathrm{eV}$.

$5.83 \mathrm{eV}$ (2 LO). For the Co-implanted AlN, the band edge transition at $6.05 \mathrm{eV}$ is absent. However, an emission line with a peak position at $5.87 \mathrm{eV}$ is evident. The spectral peak position and the intensity of the peak suggest that point defect recombination centers were created during ionimplantation. Furthermore, PL spectra of $\mathrm{Mn}$ and $\mathrm{Cr}$ implanted samples (published in Ref. 3) were identical to that of the Co-implanted sample independent of the implanted species, which also suggests that the $5.87 \mathrm{eV}$ emission line is not related with the Co ions, but to the defect recombination centers created during ion implantation. The band edge transition at $6.05 \mathrm{eV}$ could not be recovered by thermal annealing in nitrogen ambient up to $1325^{\circ} \mathrm{C}$.

Time-resolved PL was employed to measure the recombination lifetime of the $5.87 \mathrm{eV}$ emission line. The measurement reveals that the decay kinetics was a single exponential with a very short decay time constant $(<20 \mathrm{ps})$, which precludes the possibility for a donor-acceptor-pair (DAP) transition. Fast decay lifetime indicates that it is a band-toimpurity type rather than DAP type transition. For the bandto-impurity transitions, the decay rate is proportional to the total impurity (defect) concentration. Our experimental results thus indicate that the defect concentration is very high, which is consistent with the absence of the band edge transitions $(6.05 \mathrm{eV})$ in ion-implanted samples. In general, ion implantation generates a large number of vacancies in the crystal. In $\mathrm{GaN}$, high dose ion implantation is known to induce nitrogen vacancies that cannot be recovered completely by thermal annealing. ${ }^{11}$ Since the $\mathrm{Al}$ vacancy $\left(V_{\mathrm{Al}}\right)$ is believed to be a deep acceptor with an energy level larger than $2 \mathrm{eV}^{7}$ the possibility of $V_{\mathrm{Al}}$ being involved in the $5.87 \mathrm{eV} \mathrm{PL}$ emission can be precluded. We thus believe that Co ions with energy $250 \mathrm{keV}$ only displace nitrogen atoms to generate nitrogen vacancies $\left(V_{\mathrm{N}}\right)$ in AlN. Antisites and $\mathrm{N}$ intersitials $\left(\mathrm{N}_{i}\right)$ are energetically less favorable due to the large lattice mismatch in the covalent radii of $\mathrm{Al}$ and $\mathrm{N}$. Our results thus indicate that the $5.87 \mathrm{eV}$ emission line is a band-to-impurity

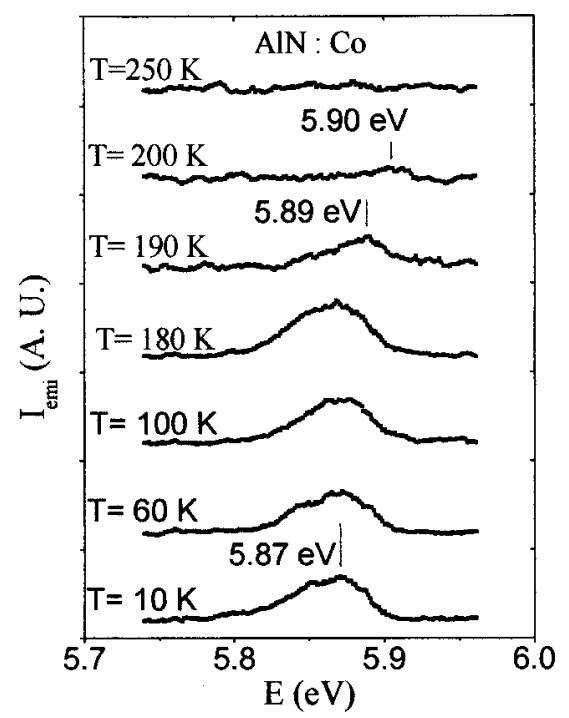

FIG. 2. PL spectra of Co-implanted AlN epilayer measured from 10 to $250 \mathrm{~K}$.

transition, involving electrons bound to $V_{\mathrm{N}}$ and free holes. As-grown AlN epilayers do not contain $V_{\mathrm{N}}$ because of the high formation energy of $V_{\mathrm{N}}$ in AlN. ${ }^{12}$ Thus, the $V_{\mathrm{N}}$ related optical transition is absent.

Figure 2 shows the temperature dependence of the 5.87 $\mathrm{eV}$ emission line in the Co-implanted AlN measured from 10 to $250 \mathrm{~K}$. The peak position changes slightly with temperature and in fact depicts a weak blueshift with increasing temperature in the range from 10 to $180 \mathrm{~K}$. This further supports our assignment that the $5.87 \mathrm{eV}$ emission line is a band-toimpurity transition, since the spectral peak position of this type of emission is expected to follow the temperature variation of the band gap with an initial blueshift before thermal energy for impurity ionization is reached. Above $170 \mathrm{~K}$, the integrated PL intensity $\left(I_{\text {int }}\right)$ of the $5.87 \mathrm{eV}$ emission line decreases with temperature rapidly and diminishes completely above $250 \mathrm{~K}$, which based on our assignment is predominantly due to the thermal activation process of $V_{\mathrm{N}}$ :

$$
V_{E_{0}}^{0} \rightarrow V_{\mathrm{N}}^{+}+e^{-},
$$

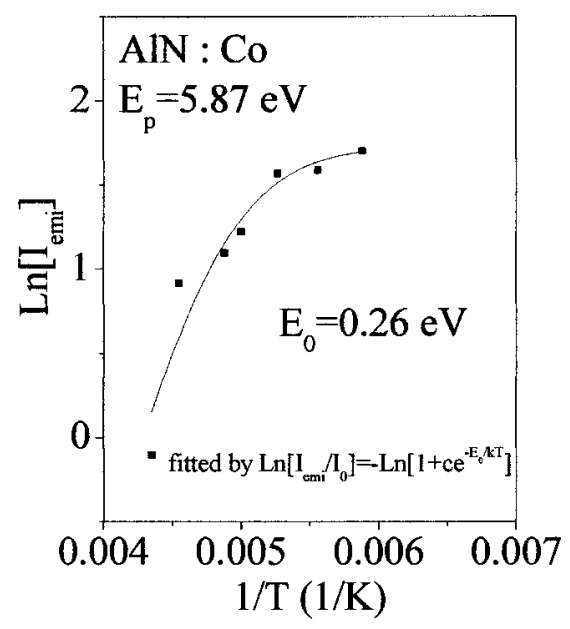

FIG. 3. The Arrhenius plot of the integrated PL emission intensity at 5.87 $\mathrm{eV}$ in the temperature range between 170 and $250 \mathrm{~K}$. The solid line is the least squares fit of data with Eq. (2). The fitted value of activation energy $\left(E_{0}\right)$ is also indicated. 


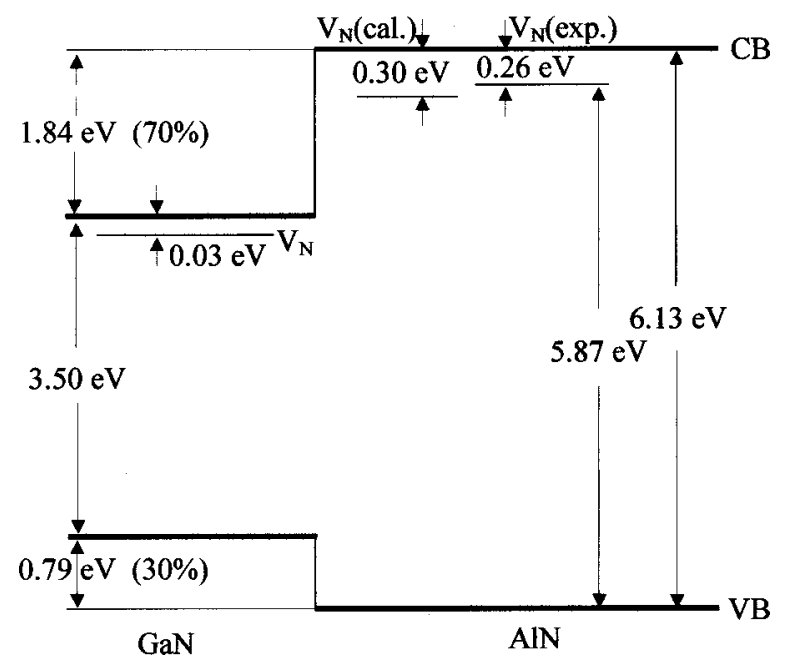

FIG. 4. The energy band diagram of AlN compared with that of GaN together with respective energy levels of $V_{N}$.

where $E_{0}$ is the thermal activation energy. Figure 3 shows the Arrhenius plot of the PL intensity of the $5.87 \mathrm{eV}$ emission line for the temperature range between 170 and $250 \mathrm{~K}$. The solid line is the least squares fit of the data with equation

$$
I_{\mathrm{emi}}=\frac{I_{0}}{1+c e^{-E_{0} / K T}},
$$

where $c$ is a constant. The fitted value of $E_{0}$ is $260 \mathrm{meV}$, which agrees quite well with the theoretically calculated energy level of $V_{\mathrm{N}}$ of $300 \mathrm{meV}^{7}$

Based on the PL results, we have constructed an energy band diagram in Fig. 4 for the $V_{\mathrm{N}}$ levels in GaN and AlN. For band alignment, a conduction-band-offset parameter of $70 \%$ between $\mathrm{AlN}$ and GaN was used ${ }^{13}$ and the energy gap of GaN was taken as $3.50 \mathrm{eV}$. Since $V_{\mathrm{N}}$ is located at $5.87 \mathrm{eV}$ with an energy level of $260 \mathrm{meV}$, the band gap of AlN can be deduced to be $5.87 \mathrm{eV}+0.26 \mathrm{eV}=6.13 \mathrm{eV}$, which agrees well with a previous determination of $6.11 \mathrm{eV} .{ }^{14}$ This, in turn, also supports our interpretation that the emission line at $5.87 \mathrm{eV}$ in Co-implanted AlN is due to a band-to-impurity transition involving $V_{\mathrm{N}}$. In contrast to GaN, the $V_{\mathrm{N}}$ in AlN cannot contribute to the $n$-type conductivity, as a consequence of the large activation energy.

In summary, MOCVD grown AIN epilayers have been implanted with Co ions and studied by deep UV PL. An emission line at $5.87 \mathrm{eV}$ has been observed, which was absent in as-grown AlN epilayers. This emission line has been attributed to a band-to-impurity transition between electrons bound to nitrogen vacancies and free holes. The experimentally determined nitrogen vacancy energy level is around 260 $\mathrm{meV}$, which is in reasonable agreement with the calculated value of $300 \mathrm{meV}$. As a consequence of the large activation energy $(0.26 \mathrm{eV})$ as well as high formation energy, $V_{\mathrm{N}}$ in AlN cannot contribute significantly to the $n$-type conductivity.

The research is supported by grants from ARO, DOE (96ER 45604/A008), and NSF (DRM-02033073), and DARPA. The authors gratefully acknowledge useful discussions with Dr. Chris Van de Walle and Professor Steve Pearton.

${ }^{1}$ Properties of Advanced Semiconductor Materials, edited by M. E. Levinshtein, S. L. Ramyantsev, and M. S. Shur (Wiley, New York, 2001), p. 31.

${ }^{2}$ V. I. Litvinov and V. K. Dugaev, Phys. Rev. Lett. 86, 5593 (2001).

${ }^{3}$ R. M. Frazier, J. Stapleton, G. T. Thaler, C. R. Abernathy, S. J. Pearton, R. Rairigh, J. Kelly, A. F. Hebard, M. L. Nakarmi, K. B. Nam, J. Y. Lin, H. X. Jiang, J. M. Zavada, and R. G. Wilson, J. Appl. Phys. 94, 1592 (2003).

${ }^{4}$ D. C. Look, D. C. Reynolds, J. W. Hemsky, J. R. Sizelove, R. L. Jones, and R. J. Molnar, Phys. Rev. Lett. 79, 2273 (1997).

${ }^{5}$ J. Neugebauer and C. G. Van de Walle, Phys. Rev. B 50, 8067 (1994).

${ }^{6}$ Q. Yang, H. Feick, and E. R. Weber, Appl. Phys. Lett. 82, 3002 (2003).

${ }^{7}$ D. W. Jenkins and J. D. Dow, Phys. Rev. B 39, 3317 (1989).

${ }^{8}$ W. J. Moore, J. A. Freitas, Jr., S. K. Lee, S. S. Park, and J. Y. Han, Phys. Rev. B 65, 081201 (2002).

${ }^{9}$ J. Li, K. B. Nam, M. L. Nakarmi, J. Y. Lin, and H. X. Jiang, Appl. Phys. Lett. 81, 3365 (2002).

${ }^{10} \mathrm{http}: / / \mathrm{www}$. phys.ksu.edu/area/GaNgroup.

${ }^{11}$ S. O. Kucheyev, J. S. Williams, C. Jaegadish, G. Li, and S. J. Pearton, J. Appl. Phys. 76, 3899 (2000).

${ }^{12}$ T. Mattila and R. M. Nieminen, Phys. Rev. B 54, 16676 (1996).

${ }^{13}$ K. B. Nam, J. Li, K. H. Kim, J. Y. Lin, and H. X. Jiang, Appl. Phys. Lett. 78, 3690 (2001).

${ }^{14}$ K. B. Nam, J. Li, M. L. Nakarmi, J. Y. Lin, and H. X. Jiang, Appl. Phys. Lett. 82, 1694 (2003). 